Jens Leonhard*

\title{
Das Präteritum im Alemannischen Südwestdeutschlands
}

https://doi.org/10.1515/zfs-2021-2028

Eingereicht 30. Juni 2020; angenommen 4. Februar 2021; vorab online veröffentlicht 8. Juli 2021

Zusammenfassung: This paper analyses the increase in the use of the preterite in spoken Alemannic in south-western Germany. There are almost no recent studies that explore the preterite in Upper German because of the widespread hypothesis that there is no preterite in Upper German (except for the verb sein 'to be') due to the loss of the preterite in Upper German (Oberdeutscher Präteritumschwund). In contrast to this, I account for a language change in the timespan from 1974 to 2013 in which the preterite becomes more frequent in relation to the perfect and is now part of the spoken Alemannic in south-western Germany. To account for this, I use a combination of a real time and an apparent time analysis. Additionally, all verbs forming a preterite have a specific semantic value, i. e. an inherent meaning of state. This means they are durative (=the situation lasts for a certain period of time), atelic (=the situation has no terminal point at which the situation is complete) and non-dynamic (=the situation involves no change). Perfect forms on the other hand do not have this specific semantic value.

Schlagwörter: tense, dialectology, preterite, perfect, lexical aspect, language change, Alemannic

\section{Einleitung}

In diesem Aufsatz untersuche ich die Zunahme der Präteritumverwendung in der gesprochenen Sprache in Teilen des Nieder-, Mittel- und Hochalemannischen (im Folgenden als Alemannisch in Baden oder Alemannisch in Südwestdeutschland abgekürzt) seit Mitte der 1970er Jahre. Jüngere Untersuchungen des Präteritums im Alemannischen Deutschlands fehlen m. W. vollständig. Der Grund dafür ist wohl die Meinung, das Präteritum sei im Alemannischen (genau wie im gesamten oberdeutschen Sprachraum) bis auf die Präteritalformen von sein ausgestorben (vgl. Rowley 1983: 164-165 und Fischer 2018: 37-39). Diese Ansicht stützt sich vor

*Korrespondenzautor: Jens Leonhard, Deutsches Seminar - Germanistische Linguistik, Albert-Ludwigs-Universität Freiburg, Freiburg, Germany, E-Mail:

jens.leonhard@germanistik.uni-freiburg.de

Ә Open Access. ( 2021 Leonhard, publiziert von De Gruyter. (cc))BY Dieses Werk ist lizensiert unter einer Creative Commons Namensnennung 4.0 International Lizenz. 
allen Dingen auf Georg Wenkers Sprachatlas des Deutschen Reichs (1888-1923) (vgl. Fischer 2018: 15) und die Ortsgrammatiken des Alemannischen Sprachraums (vgl. Rowley 1983: 164-165 und Fischer 2018: 37-39) wie beispielsweise Oechsner (1951), Mehne (1954), Burkart (1965), Baur (1967), Hufnagl (1967), Meng (1967), Frey (1975), Vogt (1977) und Braunstein (1978). Die einzige, mir bekannte, Gegenposition zur weitverbreiteten Annahme, das Präteritum sei im Alemannischen verschwunden, nimmt Gersbach (1982) ein. Er kommt bei seiner Auswertung des Zwirner-Korpus mit Tonaufnahmen freier Gespräche zu dem Schluss, dass von einem absoluten Fehlen des Präteritums im Oberdeutschen nicht (mehr) die Rede sein könne. Allerdings beschränke sich das Präteritum auf ein paar wenige Verben (Gersbach 1982: 224). Da er nicht angibt, ob die von ihm beobachteten Präteritalformen aus ländlichen Gegenden kommen, in denen traditionell standardferner gesprochen wird, oder aus größeren Orten (vgl. Gersbach 1982: 216), vermutet Rowley, es handle sich bei den von Gersbach gefundenen Präteritumformen um Belege aus dem Stadtdialekt und damit aus standardnaher Sprache (Rowley 1983: 165). Dass auch oberdeutsche Sprecher das Präteritum kennen, macht unter anderem Widén deutlich. Er verweist darauf, dass oberdeutsche Sprecher aufgrund ihrer Schulbildung das Präteritum bilden können und dieses nutzen, um vornehm zu klingen (Widén 1970: 283-284). Ich untersuche daher zunächst, ob die Präteritalformen, die in meinem Untersuchungskorpus vorkommen, in dialektaler oder in standardnaher Sprechweise geäußert werden. Da ich in dieser Arbeit das Präteritum im Alemannischen betrachten möchte, schließe ich Präteritalformen, die in standardnaher Sprechweise geäußert werden, von der weiteren Untersuchung aus.

Im Standarddeutschen kann das Perfekt im Gegensatz zum Präteritum auch die Bedeutung der gegenwartsbezogenen Vergangenheit haben. In dieser Bedeutung beschreibt das Perfekt eine Handlung, die abgeschlossen ist und deren Resultat fortbesteht (Musan 1999: 36-37; Helbig und Buscha 2001: 135; Szczepaniak 2011: 133-134 und Fischer 2018: 192). Die Bedeutung des Präteritums wird dagegen als imperfektiv (Eisenberg 1994: 121) oder durativ (Heidolph et al. 1984: 508-509) beschrieben. Da Perfekte mit Gegenwartsrelevanz nicht mit dem Präteritum austauschbar sind, werde ich diese Belege für die weitere Untersuchung ausschließen.

Im Zentrum dieses Beitrags steht die jüngere (sprachgeschichtliche) Entwicklung des Präteritums. Dabei wird die Frage behandelt, ob die Frequenz des Präteritums im Vergleich zu den Perfektformen im Untersuchungszeitraum (1974-2013) zugenommen hat. Mit einer kombinierten Real-Time-/Apparent-Time-Analyse soll diese Frage beantwortet werden. Verglichen werden dabei nur Fälle, in denen die beiden Tempora austauschbar sind. Darüber hinaus untersuche ich, von welchen 
Verben ein Präteritum gebildet wird und welche Gemeinsamkeit diese Verben aufweisen. Ich stelle hierzu kurz die lexikalisch-semantische Kategorie Aktionsart vor und zeige, dass fast alle Verben, die ein Präteritum bilden, der Aktionsart State zuzuordnen sind, $d$. h. dass sie Zustände beschreiben. Abschließend vergleiche ich die Verteilung von Perfekt und Präteritum auf die Aktionsarten und untersuche, ob die Tempora Affinitäten zu Aktionsarten aufweisen.

\section{Korpus und Methode ${ }^{1}$}

Die Grundlage der Untersuchung bilden die Erhebungen des Südwestdeutschen Sprachatlasses (SSA) sowie der Projekte REDI (Stoeckle 2014 und Hansen-Morath 2016) und FLARS (Auer et al. 2015). Die Aufnahmen entstanden in den Jahren 1974-1985 bzw. 2007-2013. Die Korpora decken also einen Untersuchungszeitraum von 39 Jahren ab.

Das erste Korpus setzt sich aus Aufnahmen des SSA zusammen. Der SSA umfasst insgesamt 579 Erhebungsorte, die in einem durchschnittlichen Abstand von $6,4 \mathrm{~km}$ über die ehemaligen Regierungsbezirke Südbaden und Südwürttemberg verteilt sind (vgl. Steger und Schupp 1993: 4). Die Gewährspersonen waren über 60 Jahre alt, im Ort geboren und aufgewachsen (vgl. Steger und Schupp 1993: 34). Auch deren Eltern sollten aus demselben Ort stammen. Pro Erhebungsort wurden mindestens zwei Gewährspersonen ausgesucht (eine Frau und ein Mann (Steger und Schupp 1993: 34)). In einer Teilmenge der Erhebungsorte wurden Tonaufnahmen, die ursprünglich für eine spätere Kontrolle der Transkription etc. gedacht waren, angefertigt. Die Aufnahmen dokumentieren teilweise Ausschnitte der Erhebungssituation, jedoch auch Konversationen unter Verwendung von Fragen ohne vorgegebene Fragestellung (vgl. Steger und Schupp 1993: 35). Für diese Untersuchung werden lediglich diese spontansprachlichen Teile und die Ortspunkte herangezogen, die im Alemannischen in Baden liegen, um eine Vergleichbarkeit mit dem zweiten, jüngeren Korpus zu gewährleisten. Daraus ergeben sich für meine Untersuchung 84 Orte. Dieses Korpus wird im Folgenden als SSA bezeichnet.

Das zweite und das dritte Korpus fußen auf den Erhebungen der Projekte REDI (2014) und FLARS (2015). Für das Projekt REDI (2014) wurden Untersuchungsorte im alemannischen Dreiländereck (Deutschland, Frankreich, Schweiz) ausgewählt (Stoeckle 2014: 99); für das Projekt FLARS (2015) wurde der gesamte Grenzverlauf des Nieder-, Mittel- und Hochalemannischen im links- und rechtsrheinischen

1 Das zusammengestellte Untersuchungskorpus wurde auch in Leonhard (2019) verwendet. Aus diesem Grund ähneln sich die jeweiligen Korpusbeschreibungen. 
Gebiet untersucht (Auer et al. 2015: 326). Für meinen Beitrag habe ich die Erhebungsorte herangezogen, die im deutschen Staatsgebiet liegen. Somit enthalten die beiden jüngeren Korpora 46 Ortspunkte. Diese Ortspunkte sind gleichmäßig über das Nieder-, Mittel- und Hochalemannische Südwestdeutschlands verteilt. Darüber hinaus handelt es sich bei diesen Orten (mit zwei minimalen Abweichungen $)^{2}$ um Orte, die bereits im Südwestdeutschen Sprachatlas untersucht wurden (vgl. Stoeckle 2014: 99-100), um einen historischen Vergleich zu ermöglichen (vgl. Auer et al. 2015: 327).

In den beiden neueren Projekten (REDI und FLARS) gibt es jeweils eine jüngere und eine ältere Gruppe von Gewährspersonen (Stoeckle 2014: 102-103). Die jüngeren Sprecher waren zum Zeitpunkt der Erhebung zwischen 25 und 35, die älteren Sprecher zwischen 60 und 70 Jahre alt. Bei den Erhebungen wurde darauf geachtet, Gewährspersonen zu gleichen Teilen aus kommunikationsorientierten und aus landwirtschaftlichen oder handwerklichen Berufen $\mathrm{zu}$ akquirieren. Da die Suche nach geeigneten Gewährspersonen in kleineren Orten zum Teil schwierig war, weicht das Alter weniger Informantinnen und Informanten minimal ab. In jedem Erhebungsort wurden mindestens zwei ältere Frauen und zwei ältere Männer sowie eine jüngere Frau und ein jüngerer Mann interviewt (Stoeckle 2014: 102; Auer et al. 2015: 327-328 und Auer et al. 2017: 28). In Erhebungsorten, die in beiden Projekten (REDI und FLARS) untersucht wurden, ergaben sich dementsprechend mindestens vier ältere Frauen und vier ältere Männer sowie zwei jüngere Frauen und zwei jüngere Männer. Aus diesen beiden Sprechergruppen ergeben sich für diese Untersuchung die zwei Korpora $R E+F L$ (alt) und $R E+F L$ (jung). Ich unterscheide in jüngere und ältere Sprecher, da laut Mattheier (1994: 427) mit dem Eintritt ins Rentenalter eine Zuwendung zu informelleren Sprechweisen und damit auch zum Dialekt hin geschieht (vgl. auch Stoeckle 2014: 103). Hansen-Morath hat im Rahmen des REDI-Projekts nachgewiesen, dass ältere Sprecherinnen und Sprecher im Durchschnitt über eine signifikant höhere Dialektkompetenz verfügen als jüngere (Hansen-Morath 2016: 46). Zwischen der jüngeren und der älteren Gruppe könnten also durchaus Unterschiede in der Verwendung der Tempora zu beobachten sein.

Die Informantinnen und Informanten sind am jeweiligen Erhebungsort geboren, beide Elternteile kommen aus dem alemannischen Sprachgebiet Deutschlands (vgl. Auer et al. 2015: 328). Durch 40 Übersetzungssätze wurde die Dialektkompetenz der Gewährspersonen getestet (vgl. Auer et al. 2015: 328). Im An-

2 Anstelle der Gemeinde Grunern wurde für FLARS (2015) das direkt angrenzende Staufen und anstelle der Freiburger Stadtteile Herdern und St. Georgen wurden die Stadtteile Betzenhausen und Lehen gewählt (Stoeckle 2014: 100). 
schluss daran führten die Exploratorinnen und Exploratoren (teilweise alleine, teilweise zu zweit) mit den Gewährspersonen ethnodialektologische Befragungen durch und stellten Fragen zu Sozialdaten und Sprachgebrauch der Informantinnen und Informanten (vgl. Stoeckle 2014: 104). In der vorliegenden Untersuchung habe ich nur die freien Gespräche berücksichtigt. Die Interviews hatten eher informellen Charakter über Dialekte, sodass die ethnodialektologischen Fragen paraphrasiert wurden (vgl. Stoeckle 2014: 105). Dadurch entstanden viele freie Gespräche und spontansprachliche Daten.

Ich behandle in dieser Arbeit ausschließlich Indikativ-Formen. Das hat zwei Gründe. Zum einen verhalten sich Konjunktiv-Formen grundsätzlich anders als Indikativ-Formen und müssen daher gesondert betrachtet werden (Thieroff 1992: 220-222). Zum anderen ist ein Vergleich von Perfekt und Präteritum nur dann möglich, wenn ausschließlich Fälle betrachtet werden, in denen die jeweiligen Tempora miteinander konkurrieren könnten. Da es im Deutschen allerdings keinen Konjunktiv I des Präteritums gibt, wird in diesen Fällen obligatorisch das Perfekt als Ersatzform gewählt (vgl. Thiel 1964: 85).

\section{Präteritum in standardnaher Sprechweise}

Wie oben dargelegt wurde, herrscht die Meinung vor, es gebe im Alemannischen höchstens ein Präteritum des Verbs sein. Im „waschechten“ (Vogt 1977: 122) Dialekt sei das Präteritum nicht gebräuchlich. Allerdings würden oberdeutsche Sprecher dieses Tempus benutzen, um besonders standardsprachlich und schriftdeutsch zu sprechen (Fischer 2018: 213). Der Wille, sich gewählt auszudrücken, führe häufig auch zu hyperkorrekten Verwendungen des Präteritums, d. h. Verwendungen mit Gegenwartsbezug (vgl. Schmeller 1821: 375; Trier 1965: 205; Hentschel und Weydt 2013: 95-96 und Fischer 2018: 213-214). Es stellt sich daher zuerst die Frage, ob die Präteritalformen, die in meinen Untersuchungskorpora zu finden sind, Teil des eigentlichen Dialekts sind oder ob diese als Code-Switching bzw. Code-Shifting vom Dialekt zum Standard zu bewerten sind. Wie oben beschrieben wurde, sind alle Gewährspersonen kompetente Dialektsprecher. Sie wechseln jedoch vereinzelt von ihrer dialektnahen in eine standardnahe Sprechweise. Diese standardnahen Sprechweisen reichen von kurzen Äußerungen bis zu kleineren oder größeren Gesprächsphasen. Ich habe nur die Äußerungen als standardnah klassifiziert, die neben möglicherweise standardnahen Präteritalformen noch weitere standardsprachliche Merkmale aufweisen. Standardnahe Präteritalformen, die in dialektaler Sprechweise geäußert werden, betrachte ich als Entlehnungen aus dem Standard. Code-Switching bzw. Code-Shifting vom 
Dialekt zum Standard kann eine kommunikative Bedeutung haben (Bürkli 1999: 288 und Schwitalla 2012: 49, Fn. 17). ${ }^{3}$

Das folgende Beispiel (1) präsentiert einen solchen Wechsel von einer standardfernen in eine standardnahe Sprechweise. Dieser plötzliche Wechsel geschieht bei einer Reformulierung (vgl. Schwitalla 2012: 49-50). Der Sprecher GP1_86 erzählt hier von seinem Heimatort Vöhrenbach.

(1) Vöhrenbach (SSA); Schulabschluss unbekannt, kommunikationsorientierter Beruf

01 GP1_86: der wo mal in vöhrebach (.) GSI isch,

02 DER [kommt] wIdda;

03 GP2_86: [ja; ]

04 GP1_86: sie sIn scho vo aMErika kumme-

05 sin scho VIEle nach amerika sie sin widda nach

vÖhrebach kumme, =

$06 \quad=j a$ des Isch_d WAHR[heit; ]

07 GP2_86: $\quad\left[j a_{-} j a\right]$ des Isch WAHR;

08 des mÜsst ma dere ZEIge,

$\rightarrow 09$ GP1_86: sie sehen also $(-)$ vöhreba wie ich vorhin schon SAgte,=

$10 \quad$ =vöhrebach ischd in d GANze welt bekAnnt worre;

Im bisherigen Gespräch erwähnte GP1_86 bereits mehrfach, dass Vöhrenbach sehr bekannt sei. In den Zeilen 01-02 erzählt GP1_86 in seiner üblichen standardfernen Sprache, dass alle, die einmal in Vöhrenbach gewesen seien, wieder dorthin zurückkämen. Er führt diese Behauptung in den Zeilen 04-06 weiter aus und berichtet davon, dass viele nach Amerika gegangen und wieder nach Vöhrenbach zurückgekehrt seien. Daraufhin leitet er in Zeile 09 eine Zusammenfassung seiner vorherigen Aussagen ein, Vöhrenbach sei weltweit bekannt. Bei dieser Äußerung in Zeile 09 handelt es sich um einen metakommunikativen Verweis auf vorangegangene Teile des Gesprächs. Während er seine restliche Erzählung dialektal äußert, wechselt er für den diskursiven Verweis in den Standard. Das ist zum einen daran zu erkennen, dass „schon“ (Z. 09) anders als in den Zeilen 04 und 05 sowie „sehen“ (Z. 09) nicht reduziert wurden. Zum anderen handelt es sich bei „vorhin“ (Z. 09) um eine standardsprachliche Variante des dialektalen Ausdrucks ebbe, den GP1_86 an anderer Stelle des Gesprächs benutzt. Die Zu-

3 Für eine genauere Darstellung der kommunikativen Bedeutung des Code-Switchings bzw. Code-Shiftings vom Dialekt zum Standard siehe Schwitalla (2012). 
sammenfassung (,vöhrebach ischd in d GANze welt bekAnnt worre“) äußert er wieder in seiner üblichen standardfernen Sprache.

Da diese Verwendungen des Präteritums nicht Teil des Dialekts sind, schließe ich sie von der weiteren Untersuchung aus. Im Folgenden werden daher nur Präteritumbelege untersucht, die in dialektaler Sprechweise geäußert werden.

\section{Zunahme der Präteritalformen in der Zeit}

Ich möchte nun in real time und in apparent time untersuchen, ob die Frequenz der Präteritalformen gegenüber den Perfektformen für die Verben zugenommen hat. Dazu sollen die Korpora SSA sowie RE+FL (alt) und RE+FL (jung) verglichen werden. Zum Vergleich werden diejenigen Verben herangezogen, deren Präteritum in mindestens einem der Korpora mindestens zweimal vorkommt. Hierbei sind zum Teil verschiedene Präfixderivationen und Partikelverbbildungen zu demselben Verb(stamm) zusammengefasst. Dabei durften sich die Präfixderivationen und Partikelverbbildungen in der Grundbedeutung nicht vom Stamm unterscheiden und mussten dieselbe Aktionsart wie der Stamm haben. So werden beispielsweise die Verben heimkommen und hinkommen, die im Duden als einzelne Verben gelistet sind (Duden 2017: 538 und 549), zum Verb kommen gezählt, da sie die Grundbedeutung von kommen lediglich spezifizieren, aber nicht grundlegend ändern. Im Gegensatz dazu bewerte ich das Verb aussehen als eigenes Verb, da es sich in semantischer und morpho-syntaktischer Valenz vom Verb sehen unterscheidet. Auch die Verben stehen und aufstehen werden getrennt betrachtet, da sie sich hinsichtlich der Aktionsart voneinander unterscheiden.

Tabelle 1 präsentiert die quantitative Verteilung der Präterital- und Perfektformen bei den jeweiligen Verben im SSA.

Tab. 1: Absolute und relative Häufigkeiten von Präteritum und Perfekt im Korpus SSA.

\begin{tabular}{lllllll}
\hline & \multicolumn{2}{l}{ Präteritum } & \multicolumn{2}{l}{ Perfekt } & \multicolumn{2}{l}{ Gesamt } \\
\hline aussehen & 0 & $(0 \%)$ & 14 & $(100 \%)$ & 14 & $(100 \%)$ \\
denken & 0 & $(0 \%)$ & 73 & $(100 \%)$ & 73 & $(100 \%)$ \\
finden & 0 & $(0 \%)$ & 16 & $(100 \%)$ & 16 & $(100 \%)$ \\
halten & 0 & $(0 \%)$ & 48 & $(100 \%)$ & 48 & $(100 \%)$ \\
kennen & 0 & $(0 \%)$ & 71 & $(100 \%)$ & 71 & $(100 \%)$ \\
lassen & 0 & $(0 \%)$ & 32 & $(100 \%)$ & 32 & $(100 \%)$ \\
sitzen & 0 & $(0 \%)$ & 21 & $(100 \%)$ & 21 & $(100 \%)$ \\
\hline
\end{tabular}


Tab. 1 (Fortsetzung)

\begin{tabular}{lrrrrrr}
\hline & \multicolumn{2}{c}{ Präteritum } & \multicolumn{2}{c}{ Perfekt } & \multicolumn{3}{c}{ Gesamt } \\
\hline wissen & 0 & $(0 \%)$ & 68 & $(100 \%)$ & 68 & $(100 \%)$ \\
haben & 7 & $(0,6 \%)$ & 1154 & $(99,4 \%)$ & 1161 & $(100 \%)$ \\
heißen & 2 & $(1,2 \%)$ & 167 & $(98,8 \%)$ & 169 & $(100 \%)$ \\
müssen & 6 & $(1,3 \%)$ & 466 & $(98,7 \%)$ & 472 & $(100 \%)$ \\
kommen & 10 & $(1,7 \%)$ & 593 & $(98,3 \%)$ & 603 & $(100 \%)$ \\
gehen & 6 & $(1,7 \%)$ & 348 & $(98,3 \%)$ & 354 & $(100 \%)$ \\
können & 6 & $(2,0 \%)$ & 295 & $(98,0 \%)$ & 301 & $(100 \%)$ \\
geben & 10 & $(3,0 \%)$ & 322 & $(97,0 \%)$ & 332 & $(100 \%)$ \\
stehen & 4 & $(4,7 \%)$ & 82 & $(95,3 \%)$ & 86 & $(100 \%)$ \\
dürfen & 3 & $(5,1 \%)$ & 56 & $(94,9 \%)$ & 59 & $(100 \%)$ \\
wollen & 9 & $(5,6 \%)$ & 153 & $(94,4 \%)$ & 162 & $(100 \%)$ \\
werden & 8 & $(6,8 \%)$ & 110 & $(93,2 \%)$ & 118 & $(100 \%)$ \\
liegen & 4 & $(10,8 \%)$ & 33 & $(89,2 \%)$ & 37 & $(100 \%)$ \\
sein & 1255 & $(41,4 \%)$ & 1780 & $(58,6 \%)$ & 3035 & $(100 \%)$ \\
sollen & 10 & $(41,7 \%)$ & 14 & $(58,3 \%)$ & 24 & $(100 \%)$ \\
\hline
\end{tabular}

Im Korpus SSA ist bei den meisten Verben eine sehr geringe Präteritumfrequenz festzustellen. 16 der 22 Verben weisen in weniger als 5\% Präteritum auf. Bei acht dieser Verben ist gar kein Präteritum belegt (aussehen, denken, finden, halten, kennen, lassen, sitzen und wissen). Das Präteritum der Verben dürfen (5,1\%), wollen $(5,6 \%)$, werden $(6,8 \%)$ und liegen $(10,8 \%)$ ist etwas häufiger. Lediglich bei den Verben sein (41,4\%) und sollen (41,7\%) ist das Präteritum fast so oft belegt wie das Perfekt.

In RE+FL (alt) (vgl. Tabelle 2) zeigt sich ein grundlegend anderes Bild.

Tab. 2: Absolute und relative Häufigkeiten von Präteritum und Perfekt im Korpus RE+FL (alt).

\begin{tabular}{lrrrrrr}
\hline & \multicolumn{2}{l}{ Präteritum } & \multicolumn{2}{l}{ Perfekt } & \multicolumn{2}{l}{ Gesamt } \\
\hline denken & 4 & $(3,1 \%)$ & 123 & $(96,9 \%)$ & 127 & $(100 \%)$ \\
kennen & 5 & $(7,9 \%)$ & 58 & $(92,1 \%)$ & 63 & $(100 \%)$ \\
halten & 3 & $(10,7 \%)$ & 25 & $(89,3 \%)$ & 28 & $(100 \%)$ \\
sitzen & 8 & $(10,8 \%)$ & 66 & $(89,2 \%)$ & 74 & $(100 \%)$ \\
wissen & 16 & $(13,6 \%)$ & 102 & $(86,4 \%)$ & 118 & $(100 \%)$ \\
aussehen & 1 & $(14,3 \%)$ & 6 & $(85,7 \%)$ & 7 & $(100 \%)$ \\
lassen & 4 & $(14,8 \%)$ & 23 & $(85,2 \%)$ & 27 & $(100 \%)$ \\
haben & 200 & $(17,4 \%)$ & 951 & $(82,6 \%)$ & 1151 & $(100 \%)$ \\
liegen & 4 & $(17,4 \%)$ & 19 & $(82,6 \%)$ & 23 & $(100 \%)$ \\
\hline
\end{tabular}


Tab. 2 (Fortsetzung)

\begin{tabular}{lrlrlrl}
\hline & \multicolumn{2}{l}{ Präteritum } & \multicolumn{2}{l}{ Perfekt } & Gesamt \\
\hline finden & 6 & $(17,6 \%)$ & 28 & $(82,4 \%)$ & 34 & $(100 \%)$ \\
kommen & 159 & $(21,5 \%)$ & 582 & $(78,5 \%)$ & 741 & $(100 \%)$ \\
heißen & 32 & $(23,2 \%)$ & 106 & $(76,8 \%)$ & 138 & $(100 \%)$ \\
stehen & 17 & $(24,3 \%)$ & 53 & $(75,7 \%)$ & 70 & $(100 \%)$ \\
gehen & 96 & $(24,7 \%)$ & 292 & $(75,3 \%)$ & 388 & $(100 \%)$ \\
müssen & 122 & $(31,2 \%)$ & 269 & $(68,8 \%)$ & 391 & $(100 \%)$ \\
können & 123 & $(40,7 \%)$ & 179 & $(59,3 \%)$ & 302 & $(100 \%)$ \\
werden & 105 & $(43,0 \%)$ & 139 & $(57,0 \%)$ & 244 & $(100 \%)$ \\
dürfen & 26 & $(52,0 \%)$ & 24 & $(48,0 \%)$ & 50 & $(100 \%)$ \\
geben & 158 & $(53,6 \%)$ & 137 & $(46,4 \%)$ & 295 & $(100 \%)$ \\
wollen & 203 & $(71,0 \%)$ & 83 & $(29,0 \%)$ & 286 & $(100 \%)$ \\
sollen & 28 & $(87,5 \%)$ & 4 & $(12,5 \%)$ & 32 & $(100 \%)$ \\
sein & 4474 & $(88,4 \%)$ & 585 & $(11,6 \%)$ & 5059 & $(100 \%)$ \\
\hline
\end{tabular}

Für jedes Verb sind Präteritalformen belegt. Dabei hat die Frequenz des Präteritums bei jedem Verb zugenommen. Nur bei den Verben denken (3,1\%) und kennen (7,9\%) macht das Präteritum im Vergleich zum Perfekt weniger als $10 \%$ der Belege aus. Bei den Verben dürfen (52,0\%), geben (53,6\%), wollen (71,0\%), sollen $(87,5 \%)$ und sein $(88,4 \%)$ ist das Präteritum sogar (zum Teil sehr viel) häufiger belegt als das Perfekt.

Der Apparent-Time-Vergleich zwischen der älteren und der jüngeren Sprechergruppe in den jüngeren Korpora offenbart, dass die Häufigkeit des Präteritums noch weiter zugenommen hat (vgl. Tabelle 3).

Tab. 3: Absolute und relative Häufigkeiten von Präteritum und Perfekt im Korpus RE+FL (jung).

\begin{tabular}{lrrrrrr}
\hline & \multicolumn{2}{l}{ Präteritum } & \multicolumn{2}{l}{ Perfekt } & \multicolumn{2}{c}{ Gesamt } \\
\hline halten & 0 & $(0 \%)$ & 10 & $(100 \%)$ & 10 & $(100 \%)$ \\
lassen & 0 & $(0 \%)$ & 3 & $(100 \%)$ & 3 & $(100 \%)$ \\
sitzen & 5 & $(10,0 \%)$ & 45 & $(90,0 \%)$ & 50 & $(100 \%)$ \\
denken & 12 & $(17,4 \%)$ & 57 & $(82,6 \%)$ & 69 & $(100 \%)$ \\
stehen & 4 & $(28,6 \%)$ & 10 & $(71,4 \%)$ & 14 & $(100 \%)$ \\
gehen & 48 & $(39,3 \%)$ & 74 & $(60,7 \%)$ & 122 & $(100 \%)$ \\
kommen & 78 & $(40,2 \%)$ & 116 & $(59,8 \%)$ & 194 & $(100 \%)$ \\
haben & 160 & $(40,8 \%)$ & 232 & $(59,2 \%)$ & 392 & $(100 \%)$ \\
kennen & 8 & $(42,1 \%)$ & 11 & $(57,9 \%)$ & 19 & $(100 \%)$ \\
\hline
\end{tabular}


Tab. 3 (Fortsetzung)

\begin{tabular}{lrlrrrr}
\hline & \multicolumn{2}{l}{ Präteritum } & \multicolumn{2}{l}{ Perfekt } & Gesamt \\
\hline wissen & 19 & $(54,3 \%)$ & 16 & $(45,7 \%)$ & 35 & $(100 \%)$ \\
liegen & 5 & $(55,6 \%)$ & 4 & $(44,4 \%)$ & 9 & $(100 \%)$ \\
heißen & 15 & $(62,5 \%)$ & 9 & $(37,5 \%)$ & 24 & $(100 \%)$ \\
werden & 64 & $(66,7 \%)$ & 32 & $(33,3 \%)$ & 96 & $(100 \%)$ \\
können & 61 & $(67,8 \%)$ & 29 & $(32,2 \%)$ & 90 & $(100 \%)$ \\
müssen & 57 & $(67,9 \%)$ & 27 & $(32,1 \%)$ & 84 & $(100 \%)$ \\
geben & 69 & $(71,9 \%)$ & 27 & $(28,1 \%)$ & 96 & $(100 \%)$ \\
aussehen & 3 & $(75,0 \%)$ & 1 & $(25,0 \%)$ & 4 & $(100 \%)$ \\
dürfen & 13 & $(76,5 \%)$ & 4 & $(23,5 \%)$ & 17 & $(100 \%)$ \\
finden & 40 & $(76,9 \%)$ & 12 & $(23,1 \%)$ & 52 & $(100 \%)$ \\
wollen & 73 & $(86,9 \%)$ & 11 & $(13,1 \%)$ & 84 & $(100 \%)$ \\
sollen & 8 & $(88,9 \%)$ & 1 & $(11,1 \%)$ & 9 & $(100 \%)$ \\
sein & 1728 & $(95,6 \%)$ & 80 & $(4,4 \%)$ & 1808 & $(100 \%)$ \\
\hline
\end{tabular}

Im Korpus RE+FL (jung) ist bei 13 der 22 Verben die Präteritumform häufiger belegt als die Perfektform. Die Verben halten und lassen weisen in der jüngeren Sprechergruppe keine Präteritumbelege auf. In RE+FL (alt) macht das Präteritum dagegen 10,7\% (halten) und 14,8\% (lassen) der Belege aus. Diese Abnahme der Präteritumfrequenz ist womöglich auf die geringe Belegzahl in RE+FL (jung) zurückzuführen. Für das Verb halten gibt es nur zehn Belege, für lassen nur drei.

Der Vergleich der Verbtypes zeigt zudem, dass die Anzahl der Verben, mit denen ein Präteritum gebildet wird, im Untersuchungszeitraum zugenommen hat. Zwar sind bereits im Korpus SSA bei 14 Verben Präteritumformen belegt (bei den Korpora RE+FL (alt) und RE+FL (jung) sind es 22 bzw. 20 Verben). Allerdings sind nur sechs dieser Verben im SSA häufiger als $5 \%$ im Präteritum verglichen mit dem Perfekt. Bei den Korpora RE+FL (alt) und RE+FL (jung) sind dagegen 21 bzw. 20 Verben häufiger als $5 \%$ der jeweiligen Belege im Präteritum.

Die nachfolgende Abbildung 1 verdeutlicht noch einmal die Zunahme der Präteritumformen der wichtigsten Verben zwischen den drei Korpora.

Zwischen dem Korpus SSA und dem Korpus RE+FL (alt) nimmt die relative Häufigkeit des Präteritums aller Verben deutlich zu. Der Vergleich zwischen $\mathrm{RE}+\mathrm{FL}$ (alt) und RE+FL (jung) zeigt, dass die relative Präteritumfrequenz der häufigen Verben zugenommen hat. Vor allem die Häufigkeit des Präteritums von finden $(17,6 \%$ vs. $76,9 \%)$ hat massiv zugenommen. Die Präteritumfrequenz des Verbs sein hat weniger stark zugenommen als die meisten anderen Verben. Das kann da- 
Zunahme der Präteritumverwendung in der Zeit

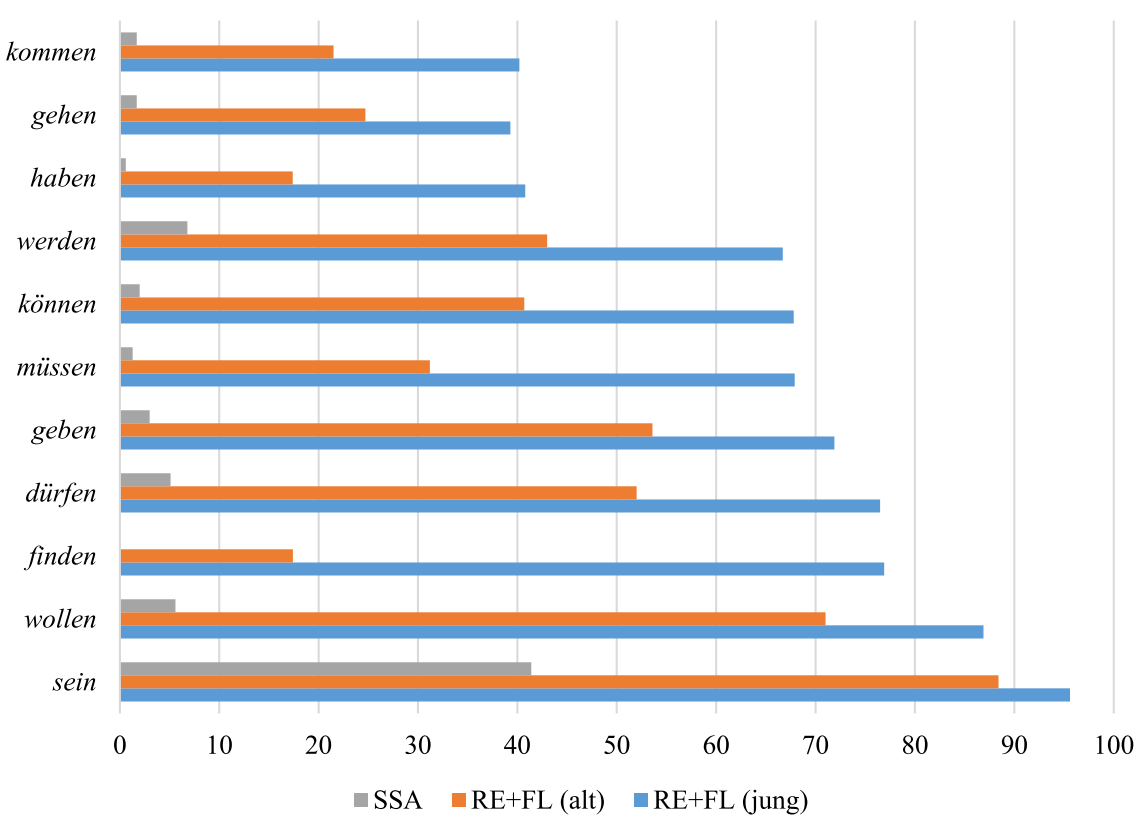

Abb. 1: Relative Häufigkeiten des Präteritums der wichtigsten Verben in den drei Teilkorpora.

mit erklärt werden, dass die Häufigkeit des Präteritums in RE+FL (alt) mit 88,4 \% bereits sehr hoch ist und eine weitere deutliche Zunahme kaum mehr möglich ist.

Darüber hinaus wurde die areale Verteilung der Präteritum-Belege untersucht. Dabei konnte zum einen eine regional gleichmäßige Zunahme der Präteritumformen im Nieder-, Mittel- und Hochalemannischen beobachtet werden. Zum anderen zeigte sich, dass die Präteritum-Belege in kleineren Orten wie Bombach (Einwohnerzahl: 660) und Holzen (Einwohnerzahl: 570) genauso vorkommen wie in größeren Orten wie Freiburg (Einwohnerzahl: 209.628) und Offenburg (Einwohnerzahl: 57.228). ${ }^{4}$

4 Für die Ermittlung der Einwohnerzahlen der Orte wurde der Zensus von 2011 herangezogen (vgl. https://www.zensus2011.de/DE/Home/home_node.html). 


\section{Formeninventar des Präteritums}

Auffällig ist, dass die meisten der 22 Verben, die im Präteritum gebraucht werden, Zustandsverben sind. Zustandsverben sind Verben der Aktionsart State. Die Aktionsart ist eine lexikalisch-semantische Verbkategorie, die den inneren zeitlichen Verlauf einer Situation festlegt. Verben lassen sich nach Vendler (1967) und Comrie (1981) in die fünf Aktionsarten State, Activity, Accomplishment, Achievement und Semelfactive unterscheiden. Für die Zuordnung konkreter Verben zu einer der Klassen können die drei binären Parameter +/-durativ, +/-dynamisch und +/-telisch herangezogen werden, die sich in ähnlicher Form bereits bei Vendler (1967: 99-121) finden und durch Comrie (1981: 41-51) exakt festgelegt wurden. Durative Verben umfassen zumindest eine gewisse Zeitdauer. Hierzu zählen States wie wissen, Activities wie essen oder Accomplishments wie bauen. Dynamisch meint, dass ein Energieaufwand aufgebracht wird oder die Veränderung einer Situation oder eines Partizipanten geschieht. Abgesehen von States sind alle Aktionsarten dynamisch. Die Telizität unterscheidet, ob Verben einen Grenzpunkt in ihrer Verbbedeutung enthalten (=telisch) oder nicht (=atelisch). Während Verben wie wissen oder essen nicht auf ein Ende gerichtet sind beziehungsweise dieses nicht in ihrer spezifischen Verbsemantik enthalten, beschreiben Verben wie finden oder aufessen singuläre Ereignisse beziehungsweise Prozesse, die ohne einen Grenzpunkt ihre eigentliche Bedeutung verlieren (Comrie 1981: 41-51). Unter States verstehe ich Verben, die eine gewisse Zeitdauer umfassen (=durativ), keinen Energieaufwand benötigen und keine Veränderung der Situation herbeiführen (=nicht-dynamisch) und keinen Grenzpunkt in ihrer Verbbedeutung enthalten (=atelisch).

Diese drei Eigenschaften treffen auf die Mehrzahl der 22 präteritumaffinen Verben zu. Zu diesen zählen die Verben aussehen, denken, haben, halten, heißen, kennen, lassen, liegen, sitzen, stehen, wissen, dürfen, können, müssen, sollen, wollen und sein.

Daneben finden sich in obiger Liste (vgl. die Tabellen 2 und 3) auch Verben, die per se keine Zustandsverben sind. Das sind finden, geben, gehen, kommen und werden. Allerdings haben diese Verben (mit Ausnahme des Verbs werden) verschiedene Bedeutungen, von denen mindestens eine aktionsartlich als State zu beurteilen ist. Im Folgenden werden die stativen Teilbedeutungen der Verben finden, geben, gehen und kommen vorgestellt. Dabei sind sehr ähnliche Bedeutungen zusammengefasst. Grundlegend dafür war, dass die Bedeutungen sich nur minimal voneinander unterscheiden und derselben Aktionsart zuzuordnen sind. Dabei wird aufgezeigt, dass die meisten Präteritalverwendungen dieser Verben States sind, d.h. Zustände beschreiben. 


\section{finden}

Beim Verb finden lassen sich im Wesentlichen zwei Bedeutungen unterscheiden: zum einen die Bedeutung '(nach einer Suche) auf etwas treffen oder stoßen' („Nach längerer Suche finde ich das Haus“) und zum anderen die Bedeutung 'etwas auf eine bestimmte Weise beurteilen oder empfinden' (,Ich finde das Haus schön“). Die erste Bedeutung ist als Aktion ${ }^{5}$ zu beurteilen, die zweite Bedeutung als Zustand (=Aktionsart State).

In Beispiel (2) erklärt die Informantin, dass sie den ersten Teil der Befragung (die Wortabfrage) als sehr interessant empfunden hat.

(2) Herrischried (RE+FL (jung)); Mittlere Reife, kommunikationsorientierter Beruf

$\rightarrow 01$ GP1_1164: abber I $x$ fAnd_s etz ebe also des des vorhin de erschde teil den fand ${ }^{x} x$ eigentlich echt INdressant ${ }^{6}$

Das Verb finden hat hier nicht die Bedeutung '(nach einer Suche) auf etwas treffen oder stoßen' („Ich suchte stundenlang, bis ich die Schlüssel fand“), sondern die Bedeutung 'etwas auf eine bestimmte Weise beurteilen oder empfinden' („Ich fand die Schlüssel sehr schön“). Im Gegensatz zur Bedeutung 'auf etwas treffen oder stoßen' ist das Verb finden hier um eine obligatorische adverbiale Gruppe (,echt INdressant") erweitert. Dementsprechend lassen sich bei allen Verwendungen des Verbs finden mit dieser Bedeutung solche Prädikativergänzungen feststellen. Die Bedeutung 'etwas einschätzen' ist als State zu interpretieren: Die hier ausgedrückte Situation ist andauernd (=durativ), es wird keine Veränderung herbeigeführt (=nicht-dynamisch) und sie ist nicht auf ein Ziel hin ausgerichtet (=atelisch).

Der quantitative Vergleich der Präteritalformen zeigt, dass alle 46 Belege des Verbs finden im Präteritum die Bedeutung 'etwas einschätzen' haben und somit als States (d.h. Zustände) zu beurteilen sind.

\section{geben}

Beim Verb geben ist in meinem Korpus neben der Hauptbedeutung 'jemandem etwas überreichen' (,Ich gebe dir das Buch“) unter anderem die Bedeutung 'existieren' („Es gab viele Bücher in der Bücherei“) auszumachen. Bei der ersten Bedeutung handelt es sich um eine Aktion, bei der zweiten um einen Zustand.

5 Aktion ist hier ein Oberbegriff für alle Aktionsarten, die keine States sind.

6 Die Sprecherin bildet bei dem Wort ich den stimmlosen uvularen Frikativ [ $]$ ] anstelle des standardnahen stimmlosen palatalen Frikativs [ç]. 
In Beispiel (3) erzählt die Gewährsperson GP1_629 von einem stadtbekannten Dichter in ihrem Heimatort Ihringen.

(3) Ihringen (SSA); Schulabschluss unbekannt, kommunikationsorientierter Beruf

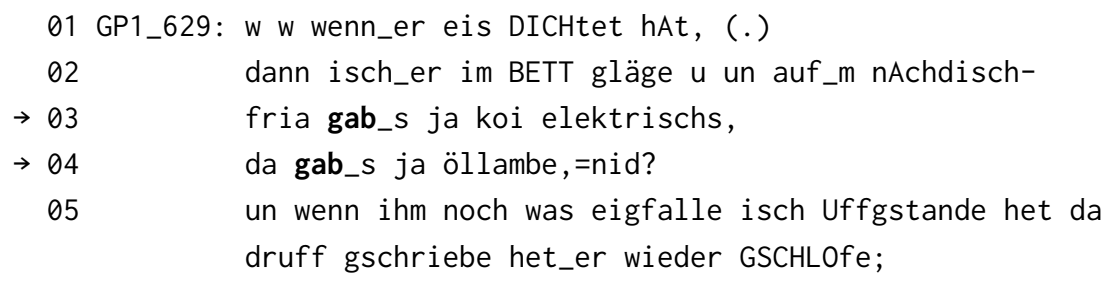

Dieser Dichter sei nachts häufig aufgestanden, um Gedichte aufzuschreiben (Z. 05). GP1_629 erwähnt bei seiner Erzählung, es habe zu dieser Zeit kein elektrisches Licht (Z. 03), sondern nur Öllampen gegeben (Z. 04).

Das Verb geben hat hier die Bedeutung 'existieren'. Formal lässt sich ein Unterschied zur Bedeutung ‘jemandem etwas überreichen’ an dem Expletivum (,es“) (bzw. hier in getilgter Form „_s“ (Z. 03-04)), dem Akkusativobjekt (,koi elektrischs“ bzw. „öllambe“) und dem fehlenden Dativobjekt erkennen. Semantisch ist die Bedeutung 'existieren’ daran ersichtlich, dass hier ein Austausch mit existieren möglich ist:

(4) a. Da gab es ja Öllampen.

b. Da existierten ja Öllampen.

Aktionsartlich ist diese Bedeutung als State zu sehen, da die hier ausgedrückte Situation ohne zeitliche Begrenzung ist (=durativ), keine Veränderung herbeigeführt wird (=nicht-dynamisch) und nicht auf ein Ziel hin ausgerichtet ist (=atelisch). Beschrieben wird ein unveränderter Zustand.

Der quantitative Vergleich offenbart, dass 236 der 237 Präteritalformen des Verbs geben als State zu beurteilen sind.

\section{gehen}

In den drei Korpora findet sich für das Verb gehen neben der Bedeutung 'von a nach b bewegen' unter anderem auch die Bedeutung 'funktionieren/möglich sein'. Die Bedeutung ‘von a nach b bewegen' ist als Aktion zu bewerten, die andere Bedeutung als Zustand.

Die Sprecherin GP1_1337 berichtet in Beispiel (5) von ihren sprachlichen Gewohnheiten in ihrer Arbeit. 
(5) Freistett (RE+FL (alt)); Schulabschluss unbekannt, kommunikationsorientierter Beruf

01 GP1_1337: vor de KINder war i ja auf_m bÜro,=

$03 \mathrm{ExJ}$ :

[hm- ]

04 GP1_1337: ' hh also do hab ich_s KAUFmännische gəmAcht,=

05 =un dann wAr_i im_me BASCHDL [(.) lade, ]

06 ExJ:

[hm mh_hm, ]

07 GP1_1337: hAb äh (-) verKAUFT [(.) do, ]

08 ExJ: $\quad$ [mh_hm, ]

09 GP1_1337: ${ }^{\circ} \mathrm{h}$ je nachdemm wenn FREIstetter kUmme sin,

un_dann isch des äh kann man KENne so spr[Eche,]

11 ExJ :

$[\mathrm{ja} ;]$

$\rightarrow 12$ GP1_1337: aber wem_ma halt äh (.) ja wenn halt ANdere kumme sin

dann (.) [ging]_s halt nId;

13 ExJ :

$[\mathrm{hm}-]$

Im Vorfeld dieses Ausschnitts fragt die Exploratorin ExJ, ob sich die Sprachgewohnheiten der Gewährsperson im Laufe der Zeit verändert haben. GP1_1337 erzählt daraufhin von ihrer Arbeit in einem Bastelladen in Freistett (ab Z. 05). Wenn Freistetter dort eingekauft hätten, habe sie ihren eigenen Dialekt sprechen können (Z. 09-10). Bei Auswärtigen sei das nicht möglich gewesen (Z. 12). Das Verb gehen hat hier die Bedeutung 'möglich sein'. Morpho-syntaktisch fordert gehen hier lediglich ein Subjekt. Es wird keine Bewegung beschrieben, sondern ausgedrückt, ob etwas möglich ist. Aus diesem Grund könnte die Konstruktion „ging_s halt nId“ (Z. 12) durch war_s halt nid möglich ersetzt werden. Auch diese Bedeutung ist als State $\mathrm{zu}$ betrachten, da es sich um einen andauernden und unbegrenzten (=durativ) Zustand handelt, der keine inhärente Veränderung beinhaltet (=nichtdynamisch) und nicht auf ein Ziel gerichtet ist (=atelisch).

Ein quantitativer Vergleich der Präteritalformen des Verbs gehen zeigt, dass 112 von 150 Belegen (74,6\%) States sind. Lediglich 38 Belege (25,3\%) sind Aktionen.

\section{kommen}

Die Grundbedeutung des Verbs kommen ('von a nach b gelangen') beschreibt eine Aktion. Als stative Bedeutungen finden sich die Bedeutungen 'irgendwo sein' und 'seinen Grund/Ursprung in etwas haben'. In Beispiel (6) berichtet der Sprecher GP1_1112 von seinem Urlaub an der Nordsee. 
(6) Bötzingen (RE+FL (alt)); Hauptschulabschluss, landwirtschaftlicher/handwerklicher Beruf

01 GP1_1112: aber wo er dann widda de DURCHsage gmAcht het,

02 nÄgschda HALdepunkt sowieso un sowieso- (1.5)

03 hab_ich (.) proBLEme khabt mit dem mit dem mit dem ostfrIEseblatt da ja;

$04 \quad$ [des isch en gAnz-]

05 EX1_1052: [hat der des ] auch RICHtig gesprochen,

06 GP1_1112: BITte?

07 EX1_1052: der hat des auch richtig [gesprochen-]

08 GP1_1112: [des des ] HAT er dann d0-

$\rightarrow 09$ die dUrchsage kame dann [ebbe] auf BLATT da unde;

10 EX1_1052: [ja:;]

Im Vorfeld dieses Ausschnitts erzählt GP1_1112, er sei mit einem Reisebus an die Nordsee gefahren. Dort habe er sich mit dem aus Norddeutschland stammenden Busfahrer unterhalten und ihn gut verstanden. Die Durchsagen des Busfahrers seien allerdings auf Plattdeutsch gewesen, sodass er ihn nur noch schwer verstanden habe (Z. 01-03). Kommen in Zeile 09 drückt keine dynamische Verbalhandlung aus, sondern einen Zustand. Das ist morpho-syntaktisch an den fehlenden Adverbialergänzungen des Ursprungs und des Ziels zu erkennen. Semantisch lässt sich der Zustand durch die Austauschbarkeit des Verbs kommen durch sein feststellen:

a. Die Durchsagen kamen auf Platt.

b. Die Durchsagen waren auf Platt.

Aus diesem Grund ist diese Verbbedeutung als durativ, dynamisch und atelisch $\mathrm{zu}$ betrachten und damit aktionsartlich als State.

Die Sprecherin GP1_1324 schildert in Beispiel (8) einen Aufenthalt in Köln, bei dem sie als Kind Verständigungsschwierigkeiten hatte.

(8) Auenheim (RE+FL (alt)); Schulabschluss unbekannt, landwirtschaftlicher/handwerklicher Beruf

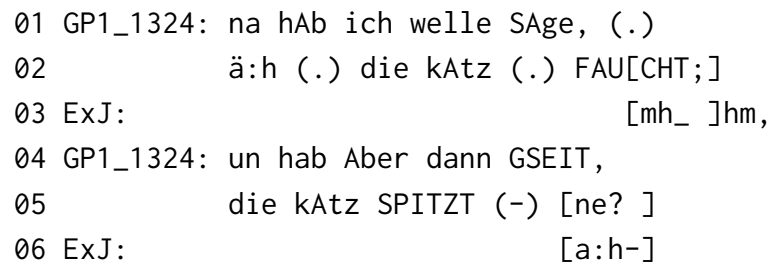




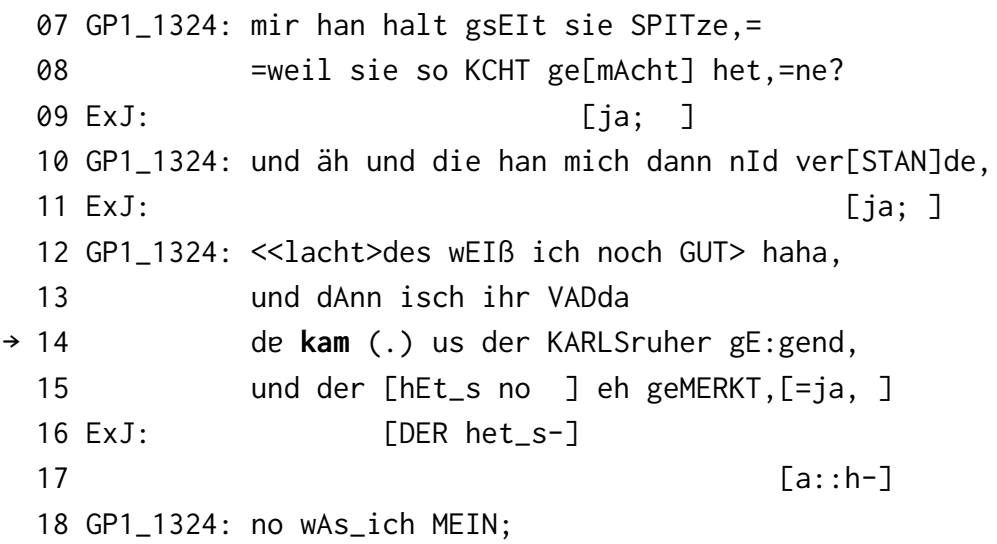

In den Zeilen 01-05 berichtet GP1_1324, sie habe bei ihrem Besuch in Köln anstelle des standardsprachlichen Ausdrucks „FAUCHT“ (Z. 02) die niederalemannische Bezeichnung „SPITZT“ (Z. 05) verwendet. Sie expandiert diese Äußerung um eine Erklärung des dialektalen Wortes in den Zeilen 07-08. Daraufhin erzählt sie, dass sie nicht verstanden wurde (Z. 10). Lediglich der Vater einer Freundin habe sie verstanden (Z. 13-18). Der Grund, warum er sie verstanden hatte, wird in Zeile 14 angegeben (,de kam (.) us der KARLSruher gE: gend“). Auch wenn eine Bewegung von einem Ort zu einem anderen mitgedacht wird (hier also von der Karlsruher Gegend nach Köln), steht hier der Ursprung der Person im Vordergrund. Das wird morpho-syntaktisch daran deutlich, dass eine die Richtung anzeigende Adverbialergänzung (nach $x$ ) fehlt und eine den Ursprung anzeigende Adverbialergänzung (,us der KARLSruher gE: gend“ (Z. 14)) obligatorisch ist. Im Gegensatz dazu ist bei der Verbbedeutung 'von a nach b gelangen' die den Ursprung anzeigende Adverbialergänzung fakultativ und die das Ziel anzeigende Adverbialergänzung obligatorisch. Semantisch wird die Ursprungsbedeutung in Beispiel (8) daran ersichtlich, dass der Ursprung als Begründung herangezogen wird. Eine Begründung für das Verständnis des Niederalemannischen wäre mit einer Bewegung nicht möglich. Aus diesem Grund könnte die Äußerung „de kam (.) us der KARLSruher gE:gend“ durch die Äußerung der war aus der Karlsruher Gegend ersetzt werden, allerdings nicht durch die Äußerung der hat sich aus der Karlsruher Gegend (hier her) bewegt. Die Bedeutung 'seinen Grund/Ursprung in etwas haben' ist dementsprechend als State zu betrachten. Es handelt sich um eine irreversible Eigenschaft, d.h. einen durativen Zustand, der keinen Energieaufwand benötigt oder eine Veränderung herbeiführt (=nicht-dynamisch) und nicht zielorientiert ist (=atelisch).

Im Gegensatz zu den Präteritalformen der Verben finden, geben und gehen sind die Präteritalformen beim Verb kommen nicht mehrheitlich Zustände. 113 von 
247 Präteritumbelegen (45,7\%) sind States, 134 von 247 Belegen (54,3\%) sind keine Zustände. Auch für das Präteritum des Verbs kommen kann eine deutliche Tendenz zu stativen Verbbedeutungen nachgewiesen werden, die allerdings weniger ausgeprägt ist als bei anderen Verben.

\section{werden}

Beim Verb werden ist dagegen keine stative Teilbedeutung festzustellen. Dieses Verb stellt allerdings insofern eine Besonderheit dar, da die meisten Präteritumbelege Hilfsverben zur Bildung des Vorgangspassivs sind. 160 der 177 Präteritumbelege in den drei Korpora sind Hilfsverben; lediglich 17 sind Kopulaverben. Eine mögliche Erklärung für diese klare Verteilung könnte in der Semantik der Passivkonstruktionen liegen: Passivbildungen ähneln semantisch den Konstruktionen mit Zustandsverb, da das Subjekt kein Agens ist und der beschriebene Vorgang nicht-dynamisch ist, d. h. keinen Energieaufwand des Partizipanten beinhaltet.

\section{Quantitative Verteilung der Aktionsarten bei Präteritum und Perfekt}

Wir konnten sehen, dass das Präteritum im Alemannischen Deutschlands fast ausschließlich von Zustandsverben und den stativen Teilbedeutungen von Aktionsverben gebildet wird. In einem zweiten Analyseschritt möchte ich nun zeigen, dass umgekehrt Zustände (also Verben mit Aktionsart State) größtenteils mit Präteritum gebildet werden. Dazu vergleiche ich zunächst die Präterital- mit den Perfektformen der Verben finden, geben, gehen und kommen und betrachte anschließend, mit welchem Tempus die jeweiligen Aktionsarten dieser Verben realisiert werden. Die nachfolgende Tabelle 4 zeigt die absoluten und relativen Häufigkeiten der Verben finden, geben, gehen und kommen im Untersuchungskorpus RE+FL (alt+jung) hinsichtlich der Perfekt-Präteritum-Verteilung.

Tab. 4: Absolute und relative Häufigkeiten von Präteritum und Perfekt der Verben finden, geben, gehen und kommen im Korpus RE+FL (alt+jung).

\begin{tabular}{lrlrrrr}
\hline & \multicolumn{2}{c}{ Präteritum } & \multicolumn{2}{c}{ Perfekt } & \multicolumn{3}{c}{ Gesamt } \\
\hline finden & 46 & $(53,5 \%)$ & 40 & $(46,5 \%)$ & 86 & $(100 \%)$ \\
geben & 227 & $(58,1 \%)$ & 164 & $(41,9 \%)$ & 391 & $(100 \%)$ \\
gehen & 144 & $(28,2 \%)$ & 366 & $(71,8 \%)$ & 510 & $(100 \%)$ \\
kommen & 237 & $(25,3 \%)$ & 698 & $(74,7 \%)$ & 935 & $(100 \%)$ \\
Gesamt & 654 & $(34,0 \%)$ & 1268 & $(66,0 \%)$ & 1922 & $(100 \%)$ \\
\hline
\end{tabular}


Das Perfekt macht 66,0 \% der Belege aus. Zwischen den einzelnen Verben gibt es zum Teil große Unterschiede in der Perfekt-Präteritum-Verteilung. Während bei den Verben finden und geben das Präteritum mit 53,5\% bzw. 58,1\% den etwas größeren Anteil aufweist, sind bei den Verben gehen und kommen nur 28,2\% bzw. 25,3\% der Belege im Präteritum.

Nachfolgend präsentiere ich die Verteilung der Tempusverwendung nach der Aktionsart für die jeweiligen Verben und anschließend für alle Verben.

Tabelle 5 zeigt die Verteilung der Aktionsarten auf Präteritum und Perfekt beim Verb finden.

Tab. 5: Absolute und relative Häufigkeiten der Aktionsarten für Präteritum und Perfekt des Verbs finden im Korpus RE+FL (alt+jung).

\begin{tabular}{lrrrrrr}
\hline & \multicolumn{2}{c}{ Präteritum } & \multicolumn{2}{c}{ Perfekt } & \multicolumn{2}{l}{ Gesamt } \\
\hline Achievement & 0 & $(0 \%)$ & 31 & $(100 \%)$ & 31 & $(100 \%)$ \\
State & 46 & $(83,6 \%)$ & 9 & $(16,4 \%)$ & 55 & $(100 \%)$ \\
Gesamt & 46 & $(53,5 \%)$ & 40 & $(46,5 \%)$ & 86 & $(100 \%)$ \\
\hline
\end{tabular}

Alle Präteritumformen des Verbs finden sind States. Beim Perfekt dagegen sind nur 22,5\% (9 der 40 Belege) States. Die Aktionsart State wird in 83,6\% der Fälle durch Präteritum ausgedrückt. Wir sehen also beim Verb finden für die Verwendung von Perfekt und Präteritum eine Spezialisierung hinsichtlich der Aktionsart.

Beim Verb geben zeigt sich ein ähnliches Bild (vgl. Tabelle 6).

Tab. 6: Absolute und relative Häufigkeiten der Aktionsarten für Präteritum und Perfekt des Verbs geben im Korpus RE+FL (alt+jung).

\begin{tabular}{lrrrrrr}
\hline & \multicolumn{2}{l}{ Präteritum } & \multicolumn{2}{l}{ Perfekt } & \multicolumn{3}{c}{ Gesamt } \\
\hline Activity & 0 & $(0 \%)$ & 14 & $(100 \%)$ & 14 & $(100 \%)$ \\
Accomplishment & 0 & $(0 \%)$ & 2 & $(100 \%)$ & 2 & $(100 \%)$ \\
Achievement & 1 & $(1,9 \%)$ & 51 & $(98,1 \%)$ & 52 & $(100 \%)$ \\
State & 226 & $(70,0 \%)$ & 97 & $(30,0 \%)$ & 323 & $(100 \%)$ \\
Gesamt & 227 & $(58,1 \%)$ & 164 & $(41,9 \%)$ & 391 & $(100 \%)$ \\
\hline
\end{tabular}

Die Präteritumformen von geben sind zu 99,6\% States. Beim Perfekt sind mit 97 von 164 Belegen (59,1\%) ebenfalls die Mehrzahl der Fälle States. Wie auch bei finden ist beim Verb geben eine Spezialisierung $\mathrm{zu}$ beobachten, bei der States größtenteils (d. h. in 70,0 \% der Fälle) im Präteritum gebraucht werden. 
Tabelle 7 zeigt die Verteilung für das Verb gehen.

Tab. 7: Absolute und relative Häufigkeiten der Aktionsarten für Präteritum und Perfekt des Verbs gehen im Korpus RE+FL (alt+jung).

\begin{tabular}{lrrrrrr}
\hline & \multicolumn{2}{l}{ Präteritum } & \multicolumn{2}{l}{ Perfekt } & \multicolumn{2}{l}{ Gesamt } \\
\hline Accomplishment & 27 & $(8,5 \%)$ & 290 & $(91,5 \%)$ & 317 & $(100 \%)$ \\
Achievement & 10 & $(62,5 \%)$ & 6 & $(37,5 \%)$ & 16 & $(100 \%)$ \\
State & 107 & $(60,5 \%)$ & 70 & $(39,5 \%)$ & 177 & $(100 \%)$ \\
Gesamt & 144 & $(28,2 \%)$ & 366 & $(71,8 \%)$ & 510 & $(100 \%)$ \\
\hline
\end{tabular}

Auch hier sind die Präteritum-Belege mit 107 von 144 (74,3\%) mehrheitlich States. Die Perfekt-Belege sind dagegen nur in 70 von 366 Fällen (19,1 \%) States. Die überwiegende Mehrzahl der Perfekt-Belege ist mit 79,2 \% (290 der 366 Belege) der Aktionsart Accomplishment zuzurechnen. Auffallend ist bei diesem Verb, dass die Aktionsart Achievement häufiger mit Präteritum als mit Perfekt gebildet wird $(62,5 \%$ vs. 37,5\%). Dabei handelt es sich wohl um eine lexikalische Besonderheit, da alle Achievements auf das Verb losgehen zurückzuführen sind. Die Aktionsart State wird mit 107 zu 70 (60,5 \%) in der Mehrzahl der Fälle im Präteritum gebraucht. Der Unterschied zwischen den beiden Tempora ist allerdings weniger deutlich als bei den eben vorgestellten Verben finden und geben.

Tabelle 8 zeigt die Verteilung für das Verb kommen.

Tab. 8: Absolute und relative Häufigkeiten der Aktionsarten für Präteritum und Perfekt des Verbs kommen im Korpus RE+FL (alt+jung).

\begin{tabular}{lrlrlll}
\hline & \multicolumn{2}{l}{ Präteritum } & \multicolumn{2}{l}{ Perfekt } & \multicolumn{2}{l}{ Gesamt } \\
\hline Accomplishment & 109 & $(15,8 \%)$ & 583 & $(84,2 \%)$ & 692 & $(100 \%)$ \\
Achievement & 17 & $(16,7 \%)$ & 85 & $(83,3 \%)$ & 102 & $(100 \%)$ \\
State & 111 & $(78,7 \%)$ & 30 & $(21,3 \%)$ & 141 & $(100 \%)$ \\
Gesamt & 237 & $(25,3 \%)$ & 698 & $(74,7 \%)$ & 935 & $(100 \%)$ \\
\hline
\end{tabular}

Bei den Präteritalformen des Verbs kommen machen States mit 111 von 237 Belegen 46,8 \% aus. Fast ebenso viele Präteritalformen sind der Aktionsart Accomplishment zuzuordnen (46,0\%). Bei den Perfektformen sind demgegenüber nur 30 von 698 Belegen (4,3\%) States. Accomplishments machen mit 583 von 698 Belegen (83,5\%) die große Mehrzahl aller Perfekte aus. Während Accomplishments und Achievements mit 84,2\% bzw. 83,3\% häufiger im Perfekt als im Prä- 
Tab. 9: Absolute und relative Häufigkeiten der Aktionsarten der Verben finden, geben, gehen und kommen für Präteritum und Perfekt im Korpus RE+FL (alt+jung).

\begin{tabular}{lrrrrrr}
\hline & \multicolumn{2}{l}{ Präteritum } & Perfekt & \multicolumn{3}{c}{ Gesamt } \\
\hline Activity & 0 & $(0 \%)$ & 14 & $(100 \%)$ & 14 & $(100 \%)$ \\
Accomplishment & 136 & $(13,5 \%)$ & 875 & $(86,5 \%)$ & 1011 & $(100 \%)$ \\
Achievement & 28 & $(13,9 \%)$ & 173 & $(86,1 \%)$ & 201 & $(100 \%)$ \\
State & 490 & $(70,4 \%)$ & 206 & $(29,6 \%)$ & 696 & $(100 \%)$ \\
Gesamt & 654 & $(34,0 \%)$ & 1268 & $(66,0 \%)$ & 1922 & $(100 \%)$ \\
\hline
\end{tabular}

teritum gebraucht werden, werden die meisten States $(78,7 \%)$ mit Präteritum gebildet.

Die obige Tabelle 9 stellt die Verteilung der Tempusverwendung nach der Aktionsart aller eben dargestellten Verben dar. ${ }^{7}$

Mit Ausnahme von States werden alle Aktionsarten mit relativen Häufigkeiten zwischen $86,1 \%$ und $100 \%$ überwiegend im Perfekt gebraucht. States werden dagegen in 70,4\% mit Präteritum gebildet. Eine binär logistische Regressionsanalyse für den Vergleich der Aktionsart State mit den restlichen Aktionsarten bei den beiden Tempora soll überprüfen, ob diese Verteilungen signifikant sind. Mit der Regressionsanalyse kann der Einfluss unabhängiger Variablen auf eine abhängige Variable gemessen werden. Die unabhängige Variable ist hier $A k$ tionsart (d. h. State oder eine andere Aktionsart); die abhängige Variable ist das gewählte Tempus (d. h. Perfekt oder Präteritum). Diese Regressionsanalyse ergibt einen höchstsignifikanten Wert für den Einfluss der Aktionsart State auf die Wahl des Tempus. Wenn ein Verb anstelle der Aktionsart State die Aktionsart Accomplishment hat, ist die Wahrscheinlichkeit für Perfekt anstelle von Präteritum das 14,3-Fache und bei der Aktionsart Achievement das 13,8-Fache. Für die Aktionsart Activity ergibt die binär logistische Regressionsanalyse kein signifikantes Ergebnis.

Die Aktionsart des Verbs hat also einen maßgeblichen Einfluss auf die Wahl des Tempus. Das Vorhandensein der beiden Tempora der einfachen Vergangenheit führt also zu einer Spezialisierung, bei der States mehrheitlich durch Präteritum ausgedrückt werden. Bei einigen Verben ist diese Spezialisierung stärker ausgeprägt als bei anderen. Während bei den Verben finden und kommen 83,6 \% bzw. 78,7\% der States im Präteritum gebraucht werden, sind es bei gehen 'nur' $60,5 \%$.

7 Kein Beleg hatte die Aktionsart Semelfactive. 


\section{Fazit}

Ich konnte in diesem Beitrag zeigen, dass das Präteritum bereits in den 1970er Jahren im Alemannischen in Baden verwendet wurde. Allerdings beschränkte sich die Verwendung größtenteils auf das Verb sein und vereinzelt auf Modalverben. Ein Real-Time-Vergleich des älteren Korpus SSA (1974-1985) mit dem jüngeren Korpus RE+FL (alt) (2007-2013) offenbart eine massive Zunahme der Tokenfrequenz und der Verbtypes, die Präteritum bilden. Auch der Apparent-Time-Vergleich der älteren mit der jüngeren Sprechergruppe in RE+FL zeigte bei den meisten Verben einen deutlichen Anstieg der Präteritumfrequenz. Der Sprachwandel, der durch den Real-Time-Vergleich der Korpora SSA und RE+FL (alt) ermittelt werden konnte, scheint sich auch beim Apparent-Time-Vergleich der älteren mit der jüngeren Sprechergruppe in RE+FL (jung) fortzusetzen.

Bei der Frage, welche Verben das Präteritum bilden, stellte sich heraus, dass 17 der 22 Verben, die ein Präteritum aufweisen, Zustandsverben sind. Zu diesen zählen auch die Kopula sein und die Modalverben, deren Existenz, wie eingangs erwähnt, in der Literatur bereits beschrieben wurde. Fünf der 22 Verben sind zwar per se keine Zustandsverben. Sie besitzen aber eine stative Teilbedeutung und werden hauptsächlich oder ausschließlich in dieser Teilbedeutung im Präteritum gebraucht. Das sind Bedeutungen, die von der Grundbedeutung verschieden sind und die aktionsartlich als State zu beurteilen sind. Fischer stellt einen stärkeren Präteritumerhalt bei irregulären und starken Verben fest: „Es sind häufige Verben, die zum Grundwortschatz gehören und von denen viele eine imperfektive Verbsemantik haben“ (Fischer 2018: 390; vgl. auch Harnisch 1997: 120-123 sowie Trost 2019), d. h. der Aktionsart State zuzurechnen sind. Die wieder in den Dialekt eingedrungenen Präteritalformen betreffen also genau die Verben, die besonders resistent gegenüber dem Präteritumschwund waren. Diese lassen sich daher als besonders präteritumaffin bezeichnen.

Da es im Alemannischen in Baden (genau wie im Standarddeutschen) keine grammatische Kategorie Aspekt gibt, wird die Abgeschlossenheit bzw. Unabgeschlossenheit von Verbalsituationen hauptsächlich durch die Aktionsart ausgedrückt. States haben eine imperfektive Bedeutung. Indem das Präteritum fast ausschließlich bei Zuständen gebraucht wird und umgekehrt Zustände größtenteils mit Präteritum realisiert werden, unterstützt die Tempusvariation die Distinktion unabgeschlossen/abgeschlossen. Da in vielen Fällen nur die Aktionsart einer Äußerung die abgeschlossene oder unabgeschlossene Situation markiert, übernehmen States dort eine tragende Rolle. In zukünftigen Untersuchungen sollte sich auf weitere Unterschiede zwischen beiden Tempora konzentriert werden. So stellt sich die Frage, ob auch die Lautstruktur oder pragmatische Gründe eine Rolle spielen. 
Jüngst konnte bereits nachgewiesen werden, dass auch das Plusquamperfekt im Alemannischen in Baden verwendet wird und im Vergleich zum Doppelperfekt an Häufigkeit zunimmt (vgl. Leonhard 2019). Da die Präteritalformen der Verben sein und haben im Dialekt vorhanden sind, ist die Voraussetzung für die Bildung des Plusquamperfekts erfüllt. Das Vorhandensein des Präteritums sorgt also auch für die Verwendung des Plusquamperfekts im Alemannischen in Baden. Weitere Forschungen sind notwendig, um zu klären, ob das Präteritum wieder in den Dialekt eingedrungen ist oder ob der Präteritumschwund, anders als bisher angenommen, nicht abgeschlossen war und ältere Untersuchungen (insbesondere Ortsgrammatiken) das Präteritum nur nicht erfasst haben. Sollte das Präteritum tatsächlich ins Alemannische Südwestdeutschlands zurückgekehrt sein, stellt sich auch die Frage, welchen Einfluss der Sprachkontakt mit dem Standarddeutschen auf die Präteritumverwendung hatte. In den Beispielen fiel zumindest auf, dass die Präteritumformen größtenteils standardnah waren.

Es ist anzunehmen, dass die Zunahme des Präteritums mit einer Annäherung der Sprecher an den Standard korreliert. Ein solcher Rückgang an Dialektalität im Alemannischen Südwestdeutschlands konnte in etlichen Untersuchungen festgestellt werden (vgl. u. a. Streck 2012 und Schwarz 2015). Allerdings erscheint es fraglich, ob die Präteritumverwendung alleine durch die Standardannäherung der Sprecher erklärt werden kann, da standardnahe Sprechweisen in dieser Untersuchung ausgeschlossen wurden. Weitere Untersuchungen sind daher erforderlich, um eine mögliche Korrelation zwischen Standardnähe und Präteritumgebrauch zu untersuchen. Sollten die Präteritumformen im Alemannischen aus dem Standarddeutschen entlehnt worden sein, so wäre anzunehmen, dass im kolloquialen Standarddeutschen dieselben Verben im Präteritum verwendet werden wie im Alemannischen in Baden. Dies gilt es in weiteren Untersuchungen zu eruieren.

\section{Literatur}

Auer, Peter, Julia Breuninger, Dominique Huck \& Martin Pfeiffer. 2015. Auswirkungen der Staatsgrenze auf die Sprachsituation im Oberrheingebiet (Frontière linguistique au Rhin Supérieur, FLARS). In Roland Kehrein, Alfred Lameli \& Stefan Rabanus (eds.), Regionale Variation des Deutschen. Projekte und Perspektiven, 323-348. Berlin \& Boston: De Gruyter.

Auer, Peter, Julia Breuninger \& Martin Pfeiffer. 2017. Neuere Entwicklungen des Alemannischen an der französisch-deutschen Sprachgrenze im Oberrheingebiet. In Helen Christen, Peter Gilles \& Christoph Purschke (eds.), Räume, Grenzen, Übergänge: Akten des 5. Kongresses der Internationalen Gesellschaft für Dialektologie des Deutschen (IGDD), 27-43. Stuttgart: Steiner. 
Baur, Gerhard Wolfram. 1967. Die Mundarten im nördlichen Schwarzwald. Marburg: Elwert.

Braunstein, Hermann. 1978. Der Dialekt des Dorfes Schutterwald (Ortenaukreis): Grammatik und Wortschatz. Schutterwald: Selbstverl. Braunstein.

Burkart, Hans. 1965. Laut- und Formenlehre der Mundart von Bühl-Kappelwindeck. Freiburg: Albert-Ludwigs-Universität Freiburg Examensarbeit.

Bürkli, Beatrice. 1999. Sprachvariation in einem Großbetrieb: Eine individuenzentrierte Analyse anhand sprachlicher Tagesläufe. Tübingen \& Basel: Francke.

Comrie, Bernard. 1981. Aspect: An introduction to the study of verbal aspect and related problems. 3rd edn. Cambridge: Cambridge University Press.

Duden. 2017. Die deutsche Rechtschreibung: Das umfassende Standardwerk auf der Grundlage der amtlichen Regeln. 27. Aufl. Berlin: Dudenverlag.

Eisenberg, Peter. 1994. Grundriß der deutschen Grammatik, 3. Aufl. Stuttgart \& Weimar: Metzler.

Fischer, Hanna. 2018. Präteritumschwund im Deutschen: Dokumentation und Erklärung eines Verdrängungsprozesses. Berlin \& Boston: De Gruyter.

Frey, Eberhard. 1975. Stuttgarter Schwäbisch: Laut- und Formenlehre eines Stuttgarter Idiolekts. Marburg: Elwert.

Gersbach, Bernhard. 1982. Die Vergangenheitstempora in oberdeutscher gesprochener Sprache: Formen, Vorkommen und Funktionen untersucht an Tonbandaufnahmen aus Baden-Württemberg, Bayrisch-Schwaben und Vorarlberg. Tübingen: Niemeyer.

Hansen-Morath, Sandra. 2016. Regionale und soziolinguistische Variation im alemannischen Dreiländereck: Quantitative Studien zum Dialektwandel. Freiburg: Albert-Ludwigs-Universität Freiburg Dissertation.

Harnisch, Rüdiger. 1997. Ein mitteldeutsches Tempusparadigma in textökonomischer Sicht. In Thomas Birkmann, Heinz Klingenberg, Damaris Nübling \& Elke Ronneberger-Sibold (eds.), Vergleichende germanische Philologie und Skandinavistik: Festschrift für Otmar Werner, 111-128. Tübingen: Niemeyer.

Heidolph, Karl E., Walter Flämig \& Wolfgang Motsch (eds.). 1984. Grundzüge einer deutschen Grammatik, 2. Aufl. Berlin: Akademie Verlag.

Helbig, Gerhard \& Joachim Buscha. 2001. Deutsche Grammatik: Ein Handbuch für den Ausländerunterricht. Berlin \& München: Langenscheidt.

Hentschel, Elke \& Harald Weydt. 2013. Handbuch der deutschen Grammatik, 4. Aufl. Berlin \& Boston: De Gruyter.

Hufnagl, Alfred. 1967. Laut- und Formenlehre der Mundart von Memmingen und Umgebung samt einer dialektgeographischen Übersicht des Landkreises Memmingen. München: Ludwig-Maximilians-Universität München Dissertation.

Leonhard, Jens. 2019. Doppelperfekt und Plusquamperfekt im Hoch- und Oberrheinalemannischen Südwestdeutschlands. Linguistik online 98(5). 77-97.

Mattheier, Klaus J. 1994. Varietätenzensus. Über die Möglichkeiten, die Verbreitung und Verwendung von Sprachvarietäten in Deutschland festzustellen. In Klaus J. Mattheier \& Peter Wiesinger (eds.), Dialektologie des Deutschen. Forschungsstand und Entwicklungstendenzen, 413-442. Tübingen: Niemeyer.

Mehne, Rolf. 1954. Die Mundart von Schwenningen am Neckar: Flexion, Wortbildung, Syntax, Schichtung. Tübingen: Universität Tübingen Dissertation.

Meng, François. 1967. Die Mundart von Auenheim bei Kehl. Strasbourg: Université Strasbourg Diplôme d'études supérieures.

Musan, Renate. 1999. Die Lesarten des Perfekts. Zeitschrift für Literaturwissenschaft und 
Linguistik 113. 6-51.

Oechsner, Hans-Adolf. 1951. Die Mundart des Nagold-Enzgebietes. Laute und Flexion, Abstufungen und Wandlungen. Tübingen: Eberhard Karls Universität Tübingen Dissertation.

Rowley, Anthony. 1983. Das Präteritum in den heutigen deutschen Dialekten. Zeitschrift für Dialektologie und Linguistik 50 (2). 161-182.

Schmeller, Johann A. 1821. Die Mundarten Bayerns grammatisch dargestellt. München: Thienemann.

Schwarz, Christian. 2015. Phonologischer Dialektwandel in den alemannischen Basisdialekten Südwestdeutschlands im 20. Jahrhundert: Eine empirische Untersuchung zum Vokalismus. Stuttgart: Steiner.

Schwitalla, Johannes. 2012. Gesprochenes Deutsch: Eine Einführung. 4. Aufl. Berlin: Erich Schmidt.

SSA = Südwestdeutscher Sprachatlas. 1989-2011. Hugo Steger, Eugen Gabriel \& Volker Schupp (eds.). Marburg: N. G. Elwert.

Steger, Hugo \& Volker Schupp (eds.). 1993. Einleitung zum Südwestdeutschen Sprachatlas I. Marburg: N. G. Elwert.

Stoeckle, Philipp. 2014. Subjektive Dialekträume im alemannischen Dreiländereck. Hildesheim: Olms.

Streck, Tobias. 2012. Phonologischer Wandel im Konsonantismus der alemannischen Dialekte Baden-Württembergs: Sprachatlasvergleich, Spontansprache und dialektometrische Studien. Stuttgart: Steiner.

Szczepaniak, Renata. 2011. Grammatikalisierung im Deutschen: Eine Einführung. 2. überarbeitete und erweiterte Aufl. Tübingen: Narr.

Thiel, Rudolf. 1964. Die Zeiten der Vergangenheit. Sprachpflege 13. 83-85.

Thieroff, Rolf. 1992. Das finite Verb im Deutschen: Tempus - Modus - Distanz. Tübingen: Narr. Trier, Jost. 1965. Stilistische Fragen der deutschen Gebrauchsprosa. Perfekt und Imperfekt. In Rudolf Henß \& Hugo Moser (eds.), Germanistik in Forschung und Lehre: Vorträge und Diskussionen des Germanistentages in Essen 21.-25.10.1964, 195-208. Berlin: Schmidt.

Trost, Igor. 2019. Die Vergangenheitstempora in Südthüringen und Oberfranken: Ein Plädoyer für eine Korrektur der Präteritumschwundisoglossen von Wenker und Sperschneider nach Süden. In Sebastian Kürschner, Mechthild Habermann \& Peter O. Müller (eds.): Methodik moderner Dialektforschung: Erhebung, Aufbereitung und Auswertung von Daten am Beispiel des Oberdeutschen, 335-364. Hildesheim, Zürich \& New York: Olms.

Vendler, Zeno. 1967. Linguistics in Philosophy. Ithaca, NY: Cornell University Press.

Vogt, Friedrich E. 1977. Schwäbisch in Laut und Schrift: Eine ergründende und ergötzliche Sprachlehre. Stuttgart: Steinkopf.

Wenker, Georg. 1888-1923. Sprachatlas des Deutschen Reichs. Handgezeichnetes Original von Emil Maurmann, Georg Wenker und Ferdinand Wrede. Marburg. Publiziert als Digitaler Wenker-Atlas (DiWA); www.regionalsprache.de.

Widén, Pertti. 1970. Das Präteritum in der deutschen Gegenwartssprache: Imperfekt und Perfekt aus der Sicht der Sprachkommunikation. Linguistik und Didaktik 4. 283-287. 